\title{
Prenatal Curcumin Administration Reverses Behavioral and Neurochemical Effects and Decreases iNOS and COX-2 Expressions in Ischemic Rat Pups
}

\author{
Maria Valéria Leimig Telles, ${ }^{1}$ Maria Elizabeth Pereira Nobre, ${ }^{1,2}$ Lucas Parente Alencar, \\ Keicy Parente de Siqueira, ${ }^{1}$ Ada Maria Farias Sousa Borges, ${ }^{1}$ \\ Márnya Wellysa Leite Tavares, ${ }^{1}$ Isabelle Bernardo Alves, ${ }^{1}$ Lara Soares Duarte, ${ }^{1}$ \\ Natália Kelly Rodrigues de Lacerda, ${ }^{1}$ Glaura Fernandes Teixeira de Alcântara, ${ }^{1}$ \\ Débora Amado Scerni, ${ }^{3}$ Kelly Rose Tavares Neves, ${ }^{2}$ and Glauce Socorro de Barros Viana ${ }^{1,2}$ \\ ${ }^{1}$ Faculty of Medicine Estácio de Juazeiro do Norte (FMJ), Avenida Tenente Raimundo Rocha 515, Cidade Universitária, \\ 63040-360 Juazeiro do Norte, CE, Brazil \\ ${ }^{2}$ Faculty of Medicine of the Federal University of Ceará (UFC), Rua Cel. Nunes de Melo 1127, Rodolfo Teófilo, \\ 60430-270 Fortaleza, CE, Brazil \\ ${ }^{3}$ Faculty of Medicine of the Federal University of São Paulo (UNIFESP), Rua Pedro de Toledo 669, Vila Mariana, \\ 04039-032 São Paulo, SP, Brazil
}

Correspondence should be addressed to Glauce Socorro de Barros Viana; gbviana@live.com

Received 24 August 2014; Revised 18 November 2014; Accepted 18 November 2014; Published 29 December 2014

Academic Editor: João Quevedo

Copyright (C) 2014 Maria Valéria Leimig Telles et al. This is an open access article distributed under the Creative Commons Attribution License, which permits unrestricted use, distribution, and reproduction in any medium, provided the original work is properly cited.

\begin{abstract}
The objectives were to evaluate alterations in ischemic rat pups, from dams administered with curcumin $(25 \mathrm{and} 50 \mathrm{mg} / \mathrm{kg})$. Tenday-old male pups were subjected or not (SO) to brain ischemia and reperfusion, for 1, 7, and 14 days (this last group was submitted to behavioral evaluation). After that, pups were euthanized for determinations of striatal DA and DOPAC in SO, ischemic from untreated (ICUD) or curcumin treated (ICTD) dams, as well as hippocampal immunohistochemistry assays for iNOS and COX-2 and cresyl violet staining. At the 14th postischemia day, the ICUD group showed increased locomotor activity and rearing behavior, which were reversed in ICTD animals. ICUD pups presented decreased striatal DA and DOPAC levels, relatively to SO, mainly at the 1st postischemia day, but also at the 7th and 14th days which were partially reversed in ICTD pups. A greater number of viable neurons were observed in ICTD, as related to the ICUD group. Ischemia increased iNOS and COX-2 expressions, in CA1 and CA3 areas, at the 1st, 7th, and 14th postischemia days, and these effects were minimized in ICTD pups. In conclusion, the prenatal curcumin treatment was shown to be neuroprotective where the drug anti-inflammatory and antioxidant effects probably play a role.
\end{abstract}

\section{Introduction}

Cerebral ischemia is a very common cerebrovascular disease and one leading cause of morbidity and mortality worldwide, whose complex pathology includes inflammatory events, as aggregation of inflammatory cells and upregulation of cytokines [1]. Furthermore, hypoxic-ischemic brain damage is a major cause of acute mortality and chronic neurologic morbidity, in infants and children. Much of our understanding on mechanisms of hypoxic-ischemic brain damage and potential therapeutic interventions derive from experimental models in the adult animal. It is accepted that a cerebral hypoxic-ischemic event of sufficient severity to deplete tissue energy reserves is rapidly followed by acidosis, glutamate 
excitotoxicity, generation of reactive oxygen species, and oxidative stress, followed by prolonged periods of delayed cell death and inflammation [2].

However, direct application of adult brain findings to the newborn animal is not a simple task, since the immature brain is generally considered resistant to the damaging effects of hypoxia and ischemia. At the same time, the immature brain exhibits periods of heightened sensitivity to injury, dependent upon the brain specific developmental stage [2]. Cerebral ischemia causes disturbances in a variety of cellular and molecular mechanisms, including oxidative phosphorylation, membrane function, neurotransmitter release, and free radical generation [3]. Evidences [4-6] show that inflammation and immunity play a key role in the pathophysiology of cerebral ischemia. However, despite the progress in this area, the translation of all this knowledge to new therapeutic interventions has largely failed.

Curcumin, a phenolic compound extracted from the rhizomes of Curcuma longa, is worldwide used as spice, flavoring agent, food preservative, coloring agent, and, in Asian countries, herbal medicine. Curcumin is nontoxic, even at high doses, and is classified by the FDA as "generally recognized as safe." A large body of evidence suggests that curcumin has a wide range of biological activities and potential therapeutic effects on numerous pathologic disorders, including diabetes, rheumatoid arthritis, multiple sclerosis, and cancer [7-11].

In the CNS, curcumin is reported to attenuate cognitive deficits, neuroinflammation, and plaque formation in Alzheimer's disease (AD) models and other neurodegenerative diseases as well [12-17]. Furthermore, curcumin decreased beta-amyloid plaques, delayed degradation of neurons, and decreased microglia formation, improving memory in patients with $\mathrm{AD}[18,19]$. However, another clinical trial [20] observed that although curcumin was generally well-tolerated the investigators were unable to demonstrate clinical or biochemical evidences for the efficacy of Curcumin C3 Complex in AD patients, in a 24-week placebo-controlled trial, perhaps due to the limited bioavailability of this compound.

Moreover, curcumin shows neuroprotective potential in cerebral ischemia and against excitotoxicity in retinal cultures of cerebral cortical neurons [21-25], although its efficacy has been object of controversy and its mechanism of action remains elusive. Thus, the objectives of the present study were to evaluate behavior and brain neurochemical alterations (determination of the striatal levels of dopamine and its metabolite) in ischemic pups, at different postischemia times, possibly induced by the curcumin treatments of female rats, during their gestational period up to the end of lactation. Furthermore, hippocampal immunohistochemistry for iNOS and COX-2, besides cresyl violet staining, were also performed.

\section{Material and Methods}

2.1. Drugs and Reagents. Curcumin, monoamine standards, and HPLC reagents were from Sigma-Aldrich (St. Louis, MO, USA); ketamine and xylazine were from König (Santana de
Parnaíba, São Paulo, Brazil). Antibodies for immunohistochemistry assays were from Santa Cruz Biotechnology (Dallas, TX, USA) or Merck-Millipore (Darmstadt, Germany). All other reagents were of analytical grade.

2.2. Animals and Experimental Protocol. Male and female Wistar rats from the Animal House of the Faculty of Medicine Estácio of Juazeiro do Norte, Brazil, were maintained under standard conditions and at a controlled temperature $(23 \pm$ $1^{\circ} \mathrm{C}$ ), with a $12 \mathrm{~h}$ dark $/ 12 \mathrm{~h}$ light cycle, and food and water ad libitum. Female rats (180-200 g) were mated at a ratio of 4 female to 1 male. They were separated from males, as soon as the pregnancy was assured and placed in individual cages. Then, female rats were divided into the following groups: controls (treated with distilled water) or those treated orally by gavage with curcumin, at the doses of 25 or $50 \mathrm{mg} / \mathrm{kg}$. The daily treatments were carried out from the first day of mating up to the end of lactation. Soon after birth, female pups were discarded and male pups divided into four groups: shamoperated (SO) and ischemic pups (from curcumin untreated dams-ICUD), that is, both groups from dams treated with distilled water; and two ischemic groups from curcumin treated dams (ICTD 25 and ICTD $50 \mathrm{mg} / \mathrm{kg}$ ). Furthermore, ten days after birth, male pups (18-20 g) were anesthetized with $25 \mathrm{mg} / \mathrm{kg}$, i.p., ketamine (Vetanarcol, König Laboratories, Brazil) and submitted or not to transient global brain ischemia by the occlusion of the left common carotid artery, for $15 \mathrm{~min}$, followed by 1-, 7-, and 14-day reperfusion. The sham-operated groups (SO) were submitted to the entire procedure, except for the artery occlusion. The group submitted to ischemia at the 14th day was also behaviorally evaluated. All animals were euthanized (decapitation) for dissection of striata and hippocampi and neurochemical evaluation (DA and DOPAC determinations in striata) performed at the 1st, 7 th, and 14th days after ischemia. Considering the high sensitivity of hippocampus to ischemic injury, histological and immunohistochemical assays in hippocampi were also performed, at those same periods. The study had the approval of the Animal Experimentation Committee of the Federal University of Ceará and the experiments were carried out in accordance with the current law and the NIH Guide for the Care and Use of Laboratory Animals.

2.3. Behavioral Evaluation. Open field test: this test evaluates a stimulant or depressant drug activity and may also indicate an anxiolytic action. We used an apparatus adapted for mice, since we worked with rat pups, and the experiments were performed always in the morning, by a blinded investigator. The arena was made of clear Plexiglas whose dimensions were $40 \mathrm{~cm} \times 40 \mathrm{~cm} \times 30 \mathrm{~cm}$ (length, width, height). The floor was divided into 9 quadrants of equal size. At the time of the experiment, the apparatus was indirectly illuminated by a red light. The following parameters were observed for $5 \mathrm{~min}$ : number of crossings with the four paws from one quadrant to another (this measures the locomotor spontaneous activity) and the number of rearing (stereotyped vertical exploratory movements). After each experiment the arena was cleaned with water. 
2.4. Concentrations of Striatal Dopamine (DA) and Its Metabolite (DOPAC), by HPLC. The striata from pups of all groups (SO, ICUD, ICTD 25, and ICTD 50), at the 1st, 7th, and 14th days after ischemia, were used for the preparation of $10 \%$ homogenates in $0.1 \mathrm{M}$ perchloric acid. This mixture was sonicated for $30 \mathrm{~s}$, centrifuged under $4^{\circ} \mathrm{C}$ for $15 \mathrm{~min}$, at $15,000 \mathrm{rpm}$. The supernatants were filtered in a $0.2 \mu \mathrm{m}$ pore membrane and $20 \mu \mathrm{L}$ of the filtered solution were injected into the HPLC column (Shim-Pack CLC-ODS, $25 \mathrm{~cm}$ ) for electrochemical detection (Shimadzu, model LCD-6A, Japan), with a $0.6 \mathrm{~mL} / \mathrm{min}$ flux. The mobile phase was prepared in $0.163 \mathrm{M}$ citric acid, $\mathrm{pH} 3.0$, containing $0.02 \mathrm{mM}$ EDTA and $0.69 \mathrm{mM}$ sodium octanesulfonic acid, $4 \%$ acetonitrile $(\mathrm{v} / \mathrm{v})$, and $1.7 \%$ tetrahydrofuran $(\mathrm{v} / \mathrm{v})$. Monoamine concentrations were determined by comparison to standards and the values expressed as $\mathrm{mg} / \mathrm{g}$ tissue.

2.5. Histological Study for Neuronal Viability (Cresyl Violet Staining). This is a basic stain used to visualize the cytoplasm of neurons, which contains several structures, including Nissl corpuscles made up essentially of RNA. The cresyl violet is an indicator of neuronal viability, since in the presence of neuronal lesion these corpuscles may disappear (chromatolysis), making possible the counting of remaining neurons. The ischemic pups (from either untreated or curcumin-treated mothers) as well as SO animals were euthanized, at different times after ischemia, and sections from their hippocampi and temporal cortices were placed in buffered formalin $(\mathrm{pH}$ 7.0) for $24 \mathrm{~h}$. Then, these sections were removed to a $70 \%$ ethanol solution where they stayed for at least another $24 \mathrm{~h}$. The sections were included into paraffin blocks and used for the preparation of $10 \mu$ slices. These slices were mounted in silanized slides and hydrated with xylol, followed by 100 down to $50 \%$ ethanol and distilled water $(2 \mathrm{~min})$. After that, the slides were immersed into the cresyl violet solution $(0.5 \%$ in buffered acetate, $\mathrm{pH}$ 3.8) and distilled water, followed by dehydration starting with 50 up to $100 \%$ alcohol and mounted beneath Entelan.

2.6. Immunohistochemistry Assays for COX-2 and iNOS in Hippocampi from Rat Pups, at Several Times after Ischemia. For immunohistochemistry assays, $10 \mu \mathrm{m}$ sections of hippocampi were deparaffinized, dehydrated in xylol and ethanol, and immersed in $0.1 \mathrm{M}$ citrate buffer $(\mathrm{pH} 6)$ under microwave heating $(18 \mathrm{~min})$, for antigen recovery. The sections, after cooling at room temperature for $20 \mathrm{~min}$, were washed with a phosphate buffered saline (PBS), followed by a 15 min blockade of endogenous peroxidase with a $3 \%$ $\mathrm{H}_{2} \mathrm{O}_{2}$ solution. The sections were incubated overnight (at $\left.4^{\circ} \mathrm{C}\right)$ with anti-COX-2 p65 antibody $(1: 50)$ or anti-iNOS $(1: 400)$ diluted in PBS-BSA. At the next day, the sections were washed in PBS and incubated for $30 \mathrm{~min}$ with the biotinylated rabbit antibody (anti-IgG), 1:400 dilutions in PBS-BSA. Then, the sections after washing in PBS were incubated for $30 \mathrm{~min}$, with the conjugated streptavidin peroxidase complex (ABC Vectastain complex, Vector Laboratories, Burlingame, CA, USA). After another washing with PBS, the sections were stained with $3,3^{\prime}$-diaminobenzidine-peroxide (DAB) chromophore, dehydrated and mounted in microscope slides for analyses.

2.7. Statistical Analyses. The data are presented as means \pm SEM and analyzed by one-way ANOVA followed by Newman-Keuls as the post hoc test for behavioral evaluation. For DA and DOPAC measurements at different times after ischemia, a two-way ANOVA was used followed by multiple $t$-tests. The differences were considered statistically significant at $P<0.05$.

\section{Results}

3.1. Behavioral Evaluation by the Open Field Test, 14 Days after Ischemia. Our results showed a behavioral alteration in ischemic pups from curcumin untreated dams (ICUD animals) that presented a 2-fold increase in locomotor activity, as related to the SO group. On the other hand, locomotor activity was completely restored in the ischemic pups from curcumin treated dams (ICTD animals), with both doses (Figure 1(a)). A similar profile was noticed with the rearing behavior (Figure 1(b)).

\subsection{DA and DOPAC Striatal Concentrations, at Different} Times after Ischemia, in the SO and Ischemic Pups from Curcumin Untreated (ICUD) or Treated Dams (ICTD). The data refer to DA and DOPAC concentrations in the SO and ischemic groups, analyzed at 1,7 , and 14 postischemia days. Ischemic pups from curcumin untreated dams (ICUD) presented 79, 58, and $47 \%$ reductions in DA contents, at the 1st, 7th, and 14th days after ischemia, respectively, as related to the SO group. These decreases were in great part reversed in the ICTD group, at the three periods after ischemia (Figure 2(a)). A similar profile was seen in DOPAC contents where the ICUD groups showed decreases of 58, 64, and 56\%, respectively, as related to the SO group. Data from the ICTD groups presented a recovery towards the DOPAC values of the SO group, at all three periods after ischemia (Figure 2(b)).

3.3. DOPAC/DA Ratios in the SO and Ischemic (ICUD and ICTD) Groups, at Different Postischemia Times. The DOPAC/ DA ratio increased the most in ischemic animals from the ICUD group, at the 1st postischemia day and decreased afterwards, according to the postischemic time. At the 14th postischemia day, DOPAC/DA values were similar among all groups (Table 1).

3.4. Histological Analyses: Cresyl Violet Staining. Figures 3(a), $3(\mathrm{~b})$, and 3(c) are representative photomicrographs from the CA1 and CA 3 regions of ICUD (ISC) and ICTD (ISC + C25) pups, at the 1st, 7th, and 14th postischemia days, respectively. The ICUD pup group shows alterations in the cell morphology and cellular degeneration, as well. However, the ICTD (ISC + C25) group presents cresyl violet stained neurons, with lesser alterations in the cell morphology, indicating the presence of higher number of viable neurons. 


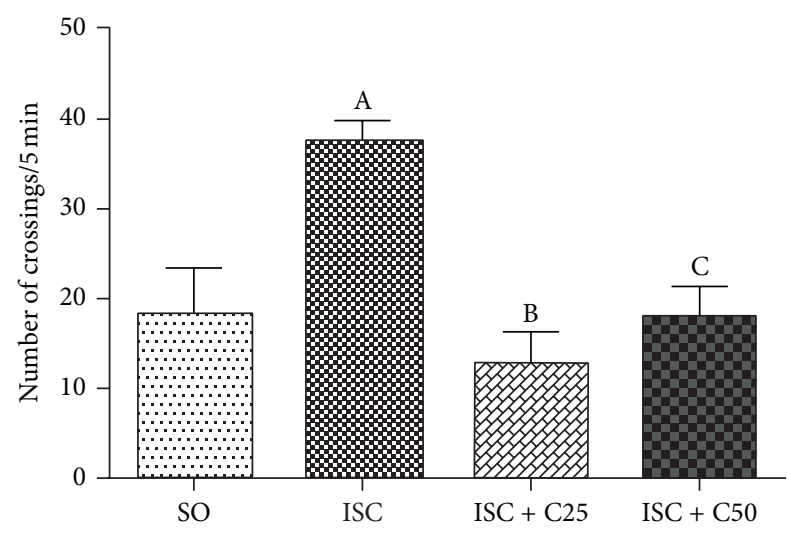

(a)

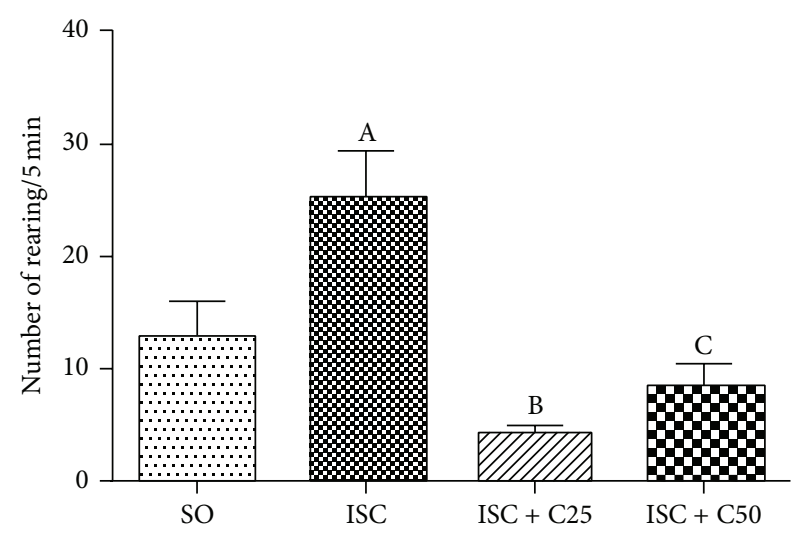

(b)

FIGURE 1: Ischemic pups from curcumin treated dams (ICTD) present decreased locomotor activity (a) and rearing behavior (b), as related to ischemic pups from curcumin untreated dams (ICUD), at the 14th day after ischemia. (a): A. versus SO, $q=5.339$; B. versus ISC, $q=6.860$; C. versus ISC, $q=5.422$. (b): A. versus SO, $q=4.397$; B. versus ISC, $q=7.098$; C. versus ISC, $q=5.984$ (one-way ANOVA and Newman-Keuls for multiple comparisons as the post hoc test).

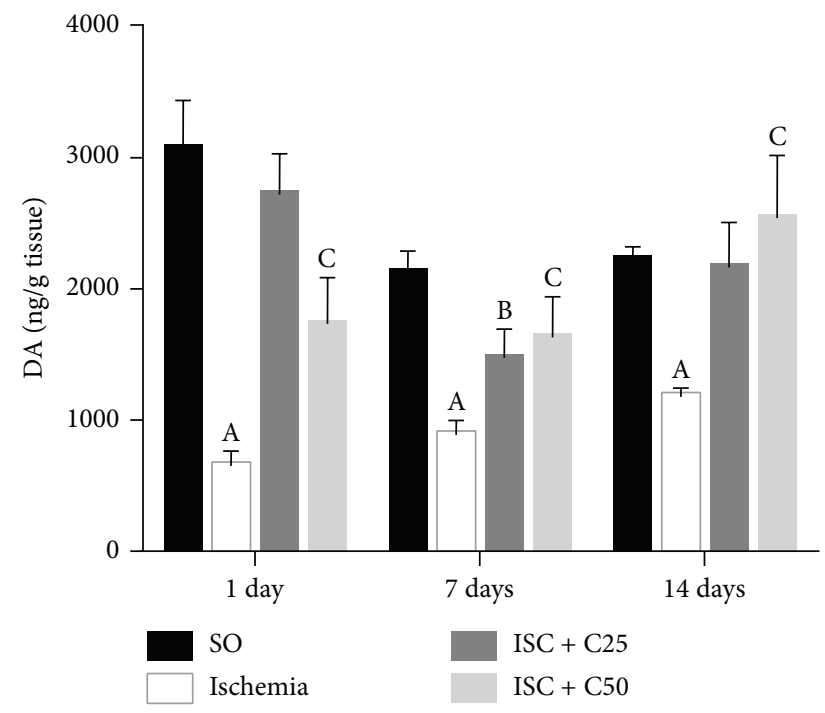

(a)

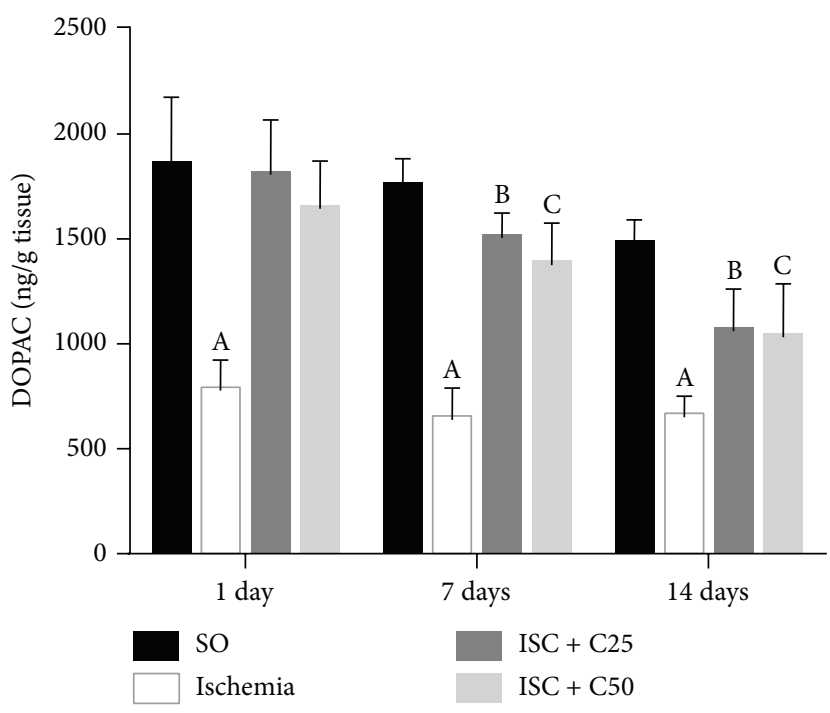

(b)

FIGURE 2: Determination of dopamine (DA) and DOPAC concentrations (ng/g tissue) by HPLC (a) and (b), in striatal tissue from 10-day-old rat pups, at the 1st, 7 th, and 14th days after the surgical procedure ( $\mathrm{SO}=$ sham-operated and ISC $=$ ischemic). The values are means $\pm \mathrm{SEM}$ from 5 to 21 animals per group. DA - A. versus SO: 1st day $(t$ ratio $=16.972, \mathrm{df}=9, P=0.0013)$; 7 th day $(t$ ratio $=17.256, \mathrm{df}=13, P=0.0001)$; 14 th day $(t$ ratio $=35.510, \mathrm{df}=31, P=0.00001)$. B. versus ISC $+\mathrm{C} 25: 7$ th day $(t$ ratio $=7.093, \mathrm{df}=16, P=0.006)$. C. versus SC $+\mathrm{C} 50: 1$ st day $(t$ ratio $=6.137, \mathrm{df}=10, P=0.0001) ; 7$ th day $(t$ ratio $=3.986, \mathrm{df}=13, P=0.0016) ; 14$ th day $(t$ ratio $=2.633, \mathrm{df}=23, P=0.0149) . \mathrm{DOPAC}$ - A. versus SO: 1 st day $(t$ ratio $=7.920, \mathrm{df}=10, P=0.009)$; 7 th day $(t$ ratio $=14.145, \mathrm{df}=12, P=0.0009) ; 14$ th day $(t$ ratio $=22.682, \mathrm{df}=32$, $P=0.0003)$. B. versus ISC + C25: 7th day $(t$ ratio $=3.846, \mathrm{df}=15, P=0.0016) ; 14$ th day $(t$ ratio $=6.736, \mathrm{df}=27, P=0.003)$. C. versus ISC + C50: 7 th day $(t$ ratio $=3.973, \mathrm{df}=12, P=0.0018 ; 14$ th day $(t$ ratio $=5.551, \mathrm{df}=20, P=0.013)$ (two-way ANOVA and multiple $t$-tests of data).

3.5. Immunohistochemical Assays for Cyclooxygenase-2 (COX2) and the Inducible Isoform Nitric Oxide Synthase (iNOS). Changes for COX-2 (Figure 4), showing an increased number of immunostained cells, were observed since the 1st day (CA1 and CA3 hippocampal areas) and continued up to the 7 th and 14th postischemia days, in the ICUD groups (ISC). These changes were reversed in the ischemic group from dams treated with curcumin (ICTD), with the dose of $25 \mathrm{mg} / \mathrm{kg}$ (ISC + C25). In the case of iNOS, changes in ischemic pups from curcumin untreated dams (ICUD) were observed at all postischemia days. Thus, while an increased number of immunostained cells were seen in CA1 and CA3 from ICUD pups, these changes were reversed in the ICTD groups (Figure 5). 

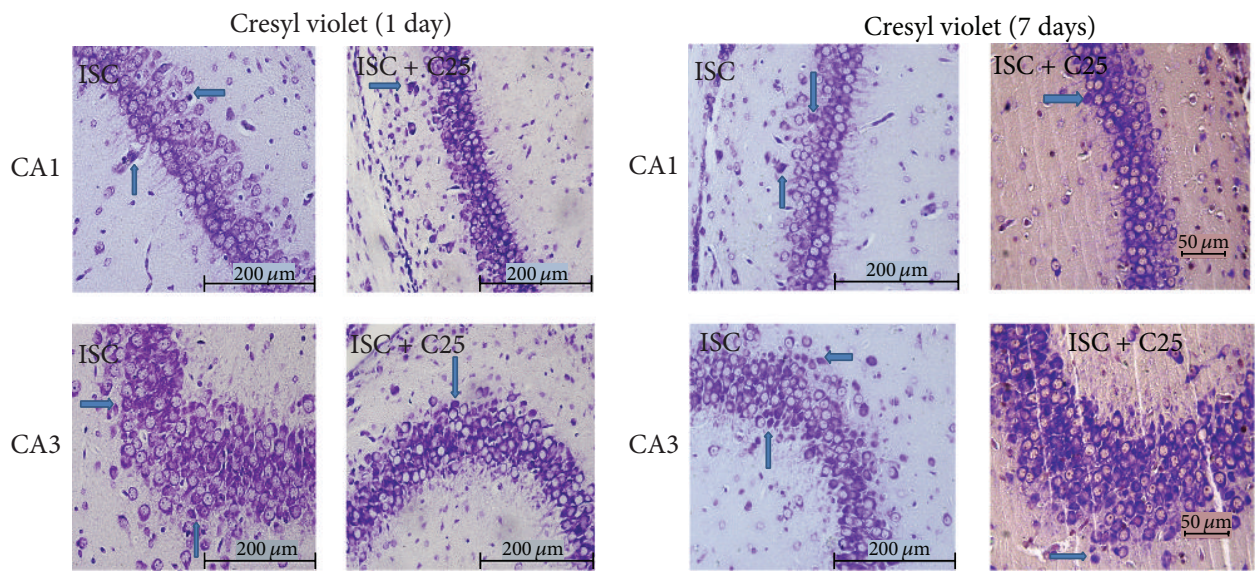

(a)

(b)

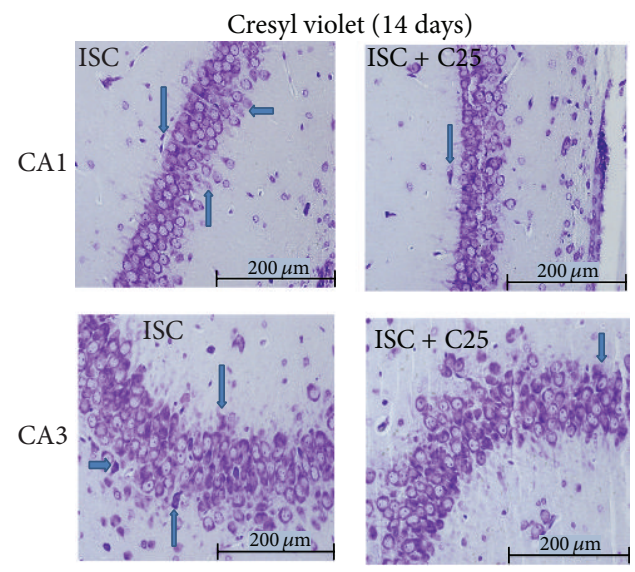

(c)

FIGURE 3: Representative cresyl violet staining photomicrographs of CA1 and CA3 hippocampal areas (a), (b), (c) from ischemic pups (ISC) of untreated (ICUD) or curcumin treated dams (ICTD) at three postischemia times. Lower numbers of viable neurons are seen in ischemic pups from untreated dams, as related to those from curcumin $(25 \mathrm{mg} / \mathrm{kg})$ treated dams (ISC + C25), at the 1st, 7th, and 14th postischemia days. The arrows indicate dark or degenerated neurons. Scale bars represent $200 \mu \mathrm{m}$ at the 1st day, 200 and $50 \mu \mathrm{m}$ at the $7 \mathrm{th}$ day, and $200 \mu \mathrm{m}$ at the 14th day after ischemia (magnification: $\times 100$ ).

\section{Discussion}

In the present work, rat pups (10 days old) from mothers treated by gavage with water or curcumin $(25$ and $50 \mathrm{mg} / \mathrm{kg}$, from mating to the end of lactation) were subjected to transient global brain ischemia. The animals were evaluated for behavioral, neurochemical, histological, and immunohistochemical alterations, at different times after ischemia. Although some studies [26] show that the unilateral carotid artery ligation alone does not induce ischemic damage, due to the intact circle of Willis, others $[27,28]$ reported that this procedure was sufficient to cause brain injury in more than $70 \%$ of 12 -day-old mice.

Although we did not carry out measurements of curcumin levels in pup brains, evidences demonstrated that the drug crosses the human blood brain barrier and is detected in the CSF [18]. Besides, curcumin in placental and foetal rodent membranes is shown to significantly reduce LPSstimulated release and gene expression of cytokines IL- 6 and to decrease COX-2 mRNA expression as well [29]. Others
TABLE 1: DOPAC/DA ratios in striatal tissues of sham-operated (SO) and ischemic rat pups (ISC) from untreated dams (ICUD) or from dams treated with curcumin (ICTD), at the doses of 25 or $50 \mathrm{mg} / \mathrm{kg}$, in different postischemia times.

\begin{tabular}{lcccc}
\hline Group & SO & ICUD & ICTD 25 & ICTD 50 \\
\hline 1st day & 0.60 & 1.19 & 0.66 & 0.95 \\
7th day & 0.82 & 0.72 & 1.02 & 0.85 \\
14th day & 0.66 & 0.55 & 0.49 & 0.41 \\
\hline
\end{tabular}

Rat pups from untreated (ICUD) or curcumin (C, 25 or $50 \mathrm{mg} / \mathrm{kg}$ ) treated dams (ICTD) were subjected to global cerebral ischemia (ISC), 10 days after birth, and euthanized for brain dissection and neurochemical determinations, at the 1st, 7 th, and 14th postischemia days. The values are from 7 to 21 animals per group.

[30] observed that curcumin (up to $10,000 \mathrm{ppm}$ ) added to the diet of female rats, though producing a small reduction in preweaning body weight gain of the second generation, did not affect (at its highest dose level) the offspring. Thus, 
CA1
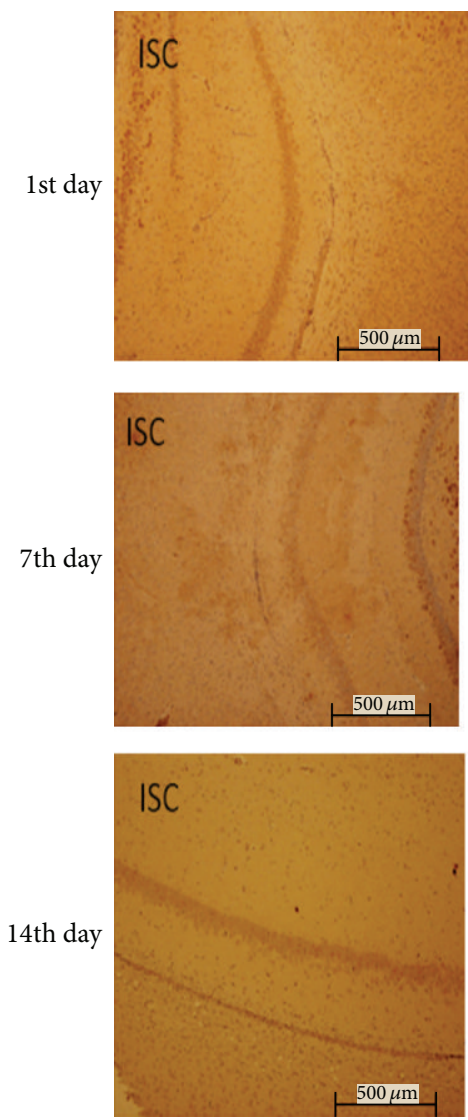
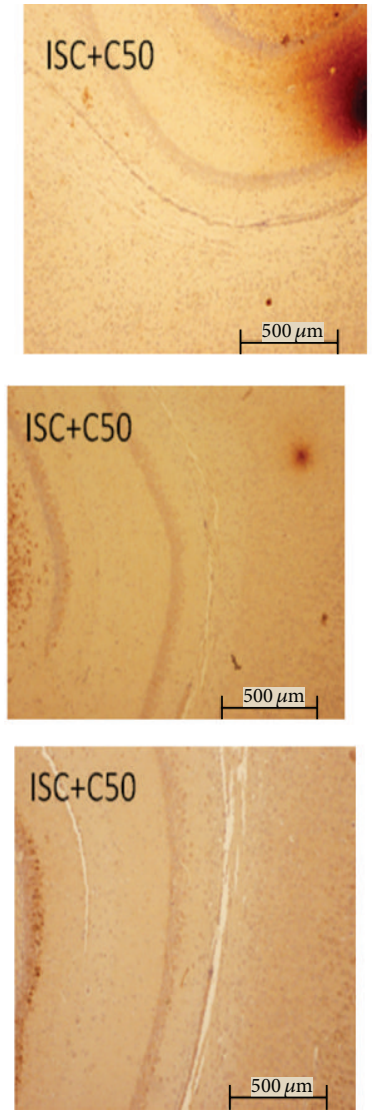

CA3
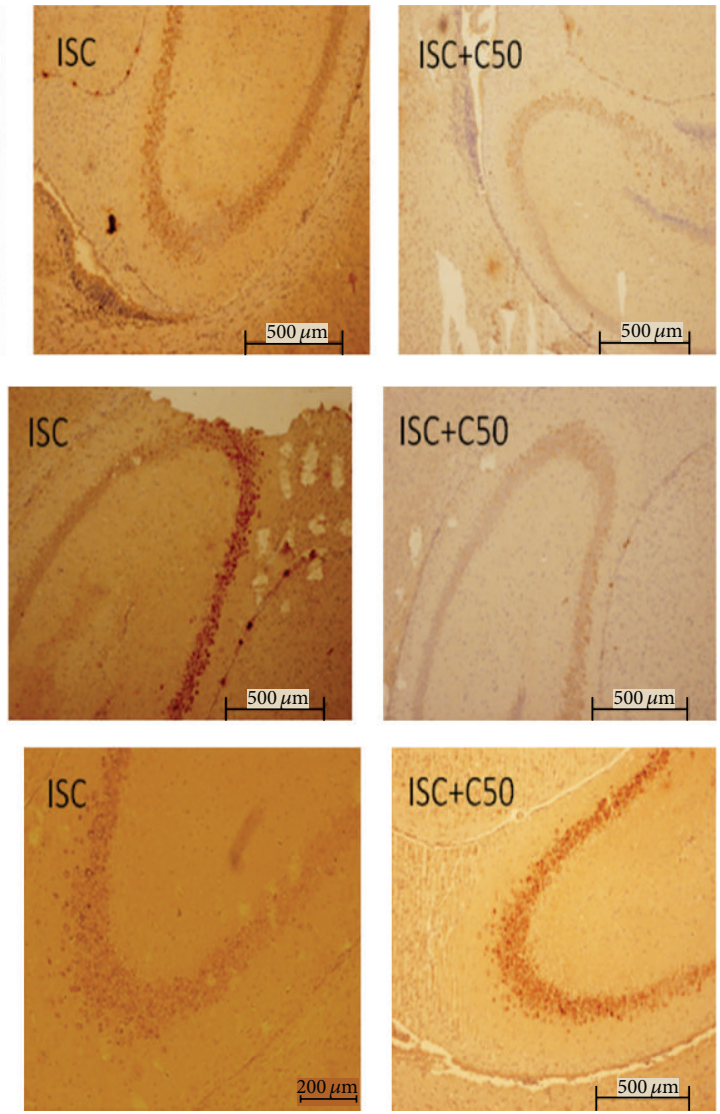

COX-2 $(\times 100)$

FIGURE 4: Representative photomicrographs of immunohistochemistry for COX-2, showing a higher number of immunostained cells in the CA1 and CA3 areas of pups from curcumin untreated dams (ICUD), at the 1st, 7th, and 14th postischemia days, as related to ischemic pups from curcumin $(25 \mathrm{mg} / \mathrm{kg})$ treated dams (ICTD). Scale bars represent 500 and $200 \mu \mathrm{m}$ (magnification $\times 100)$.

we believe that the drug reached the foetuses under our experimental conditions.

Locomotor activity is considered a useful predictor to determine the sensitivity of animals to ischemia [31]. Increased locomotor activity is found in the global ischemic model when brain ischemia is induced by the short term ( $<15$ min) occlusion of common carotids [32]. In the present work, ischemic animals showed an increased locomotor activity as well as an increased rearing behavior, at 14 days after surgery, as related to the SO group. On the hand, ischemic pups from curcumin treated dams reversed these alterations completely. Similarly to our findings, timedependent changes were observed in ischemic rats with increased locomotor activity, at 1, 5, and 30 postreperfusion days [33]. Other investigators also observed an increased locomotor activity in rats $[34,35]$ and mice [36], in hypoxiaischemia models.

Our results also showed important alterations in the dopaminergic system of pups, depending upon the postischemia time. Thus, ischemia alone caused a drastic reduction in striatal DA and DOPAC levels, mainly at the 1st postischemia day. On the other hand, changes in DA contents observed in ischemic pups from curcumin untreated dams (ICUD), at all times after ischemia, were partially or totally reversed in ischemic pups from curcumin treated dams (ICTD). Evidences [37] show increased NE but not DA contents, with a transient brain ischemia model in gerbils, and the authors concluded that increased NE but not DA plays probably a significant role in the animals' increased locomotor activity.

Striatal DA and DOPAC alterations may vary, according to the time after ischemia. Interestingly, we showed that while the DOPAC/DA ratio increases in the ICUD group, at the 1st postischemia day, it decreases at the 7th and 14th postischemia days. Although at this last time DOPAC/DA values were similar among groups (ICUD and ICTD), they were lower than those of the SO group. Almost three decades ago, Silverstein and Johnston [38] measured acute alterations in DA contents in the striatum of rat pups exposed to hypoxia-ischemia, hypoxia only, or global ischemia. When exposed to unilateral ligation of the carotid and to $2 \mathrm{~h}$ in an $8 \% \mathrm{O}_{2}$ atmosphere, the 7-day-old rat pups presented an asymmetrical rotational behavior, followed by a $70 \%$ reduction in the striatal DA levels, in the ischemic side. Although this is a 
CA1
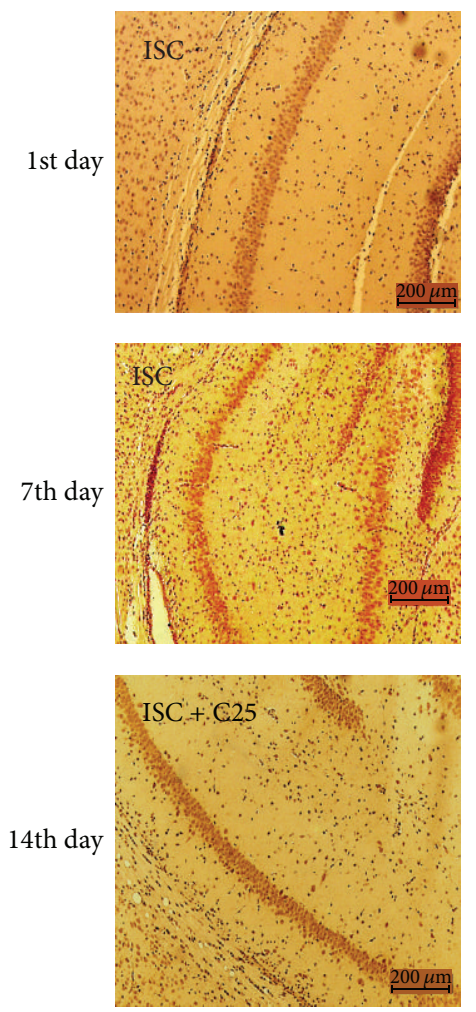
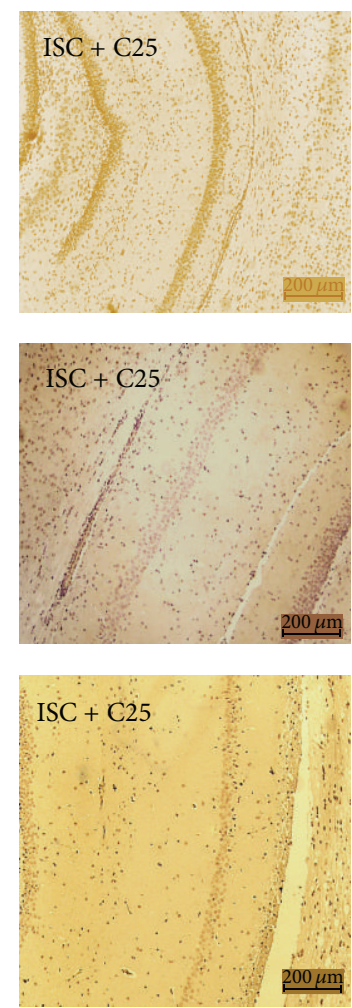

CA3
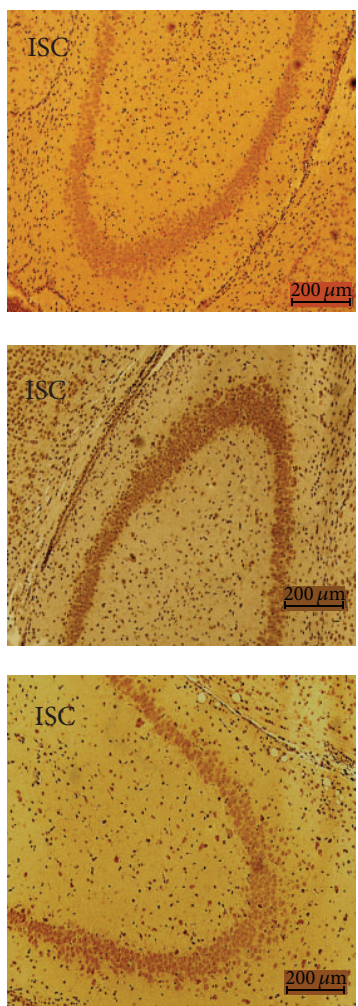

iNOS $(\times 100)$
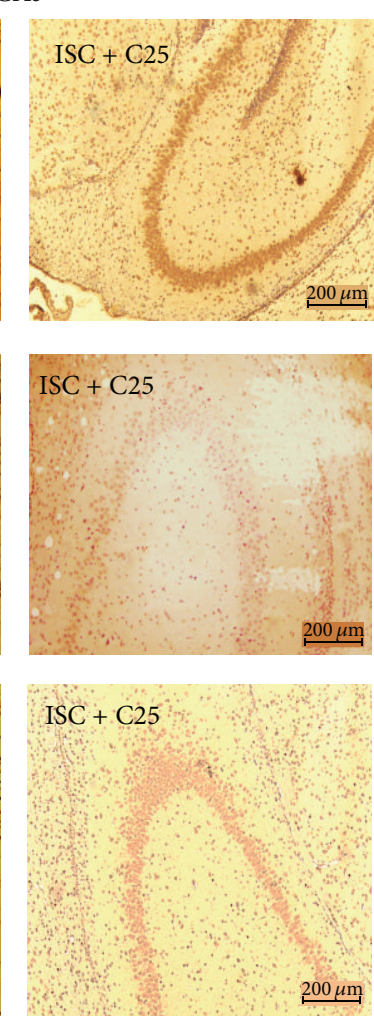

FIGURE 5: Representative photomicrographs of immunohistochemistry for iNOS, showing a higher number of immunostained cells in the CA1 and CA3 areas of ischemic (ISC) pups from untreated dams (ICUD), at the 1st, 7th, and 14th postischemia days, as related to ischemic pups from curcumin treated $(25 \mathrm{mg} / \mathrm{kg})$ dams (ICTD). Scale bars represent $200 \mu \mathrm{m}$ (magnification $\times 100)$.

pioneer work in the area, we feel that its great limitation was the absence of DOPAC determination, considering that this is the main DA metabolite in the rat brain.

Other studies, performed at the same decade [39], showed time related alterations in the striatal monoamine metabolism, after irreversible unilateral ligation of the carotid and stroke, in gerbils. These authors observed a 30\% reduction of DA content in the contralateral and nonischemic striatum, around $2 \mathrm{~h}$ after the brain insult, while DOPAC levels increased by $227 \%$. The levels of DOPAC were still higher in ischemic striata, as related to those of the nonischemic contralateral striata, at four and seven hours after stroke. After $16 \mathrm{~h}$, the levels of DA and its metabolites were depleted from the ischemic striatum, indicating irreversible brain injury and uptake of the neurotransmitter by dopaminergic nerve terminals.

In the present study, we used the model of unilateral carotid occlusion, followed by reperfusion in the absence of hypoxia, in rat pups. Under this experimental condition, we observed alterations in DA metabolism and a partial and even total reversion of the effects, by the prenatal curcumin administration. These alterations were demonstrated not only in DA and DOPAC concentrations, but also in the DOPAC/DA ratio, for ICUD as well as for ICTD groups, as related to the SO group, indicating changes in DA turnover and metabolism.
Brain ischemia is known to initiate a cascade of detrimental events, including glutamate-induced excitotoxicity, increased intracellular $\mathrm{CA}^{2+}$, production of reactive oxygen species, degradation of membrane lipids, and DNA damage. All these events lead to a rupture in the cellular homeostasis mechanisms and structural damage to the ischemic brain tissue [40]. Brain ischemia also triggers an acute inflammatory process, which exacerbates the primary brain damage.

Curcumin (100 and $300 \mathrm{mg} / \mathrm{kg}$, i.p.) was shown to decrease in a dose-dependent manner the infarcted area volume and edema, in the model of focal brain ischemia in rats [22]. Curcumin also decreased lipid peroxidation and increased superoxide dismutase (SOD) and glutathione peroxidase (GPx) activities. According to these authors, the neuroprotective action of curcumin in brain ischemia is mediated by its antioxidant activity. Furthermore, the oxidative stress is known to contribute to neurodegeneration, subsequent to ischemic cerebral injury. In this sense, AlOmar et al., 2006 [41], studied the possible antioxidant and neuroprotective actions of curcumin on neurons death, in the CA1 hippocampal area, after a process of transitory cerebral ischemia in rats. These authors observed that the curcumin treatment $(200 \mathrm{mg} / \mathrm{kg}$, i.p.), at three times (immediately and at 3 and $24 \mathrm{~h}$ after ischemia), reduced the neuronal damage observed 7 days after the ischemic event. Besides, curcumin administration to the ischemic rats decreased lipid 
peroxidation and increased the levels of glutathione (GSH), SOD, and catalase. They concluded that the curcumin treatment attenuates brain-ischemia-induced neuronal injury and hippocampal oxidative stress.

The anti-inflammatory effects of curcumin are possibly mediated by its capacity to inhibit the activity of the enzymes, as cyclooxygenase 2 (COX-2), lipoxygenase (LOX), and inducible nitric oxide synthase (iNOS), important mediators of inflammatory processes. The upregulation of COX2 and/or iNOS is associated with the pathophysiology of some human cancer types, as well as other pathologies where inflammation is an important factor [42]. Another work [43] showed that curcumin inhibited the COX-2 expression in HT-29 colon cancer cells. In this in vitro model, curcumin inhibited specifically COX-2 and did not present any effect on COX-1.

We also showed that curcumin presents a cytoprotective action in the hippocampus (CA1 and CA3 areas), as evaluated by cresyl violet staining. As related to our immunohistochemistry studies, we showed the intensity of immunostaining for iNOS and COX-2, in the ischemic pup group (from curcumin untreated dams, ICUD), to be dependent upon the time elapsed after ischemia. In general, the immunostaining for iNOS increased in hippocampal CA1 and CA3 areas, at the 1st, 7th, and 14th postischemia days, and decreased in the ischemic pup group from curcumin treated dams (ICTD). Similar results were observed for COX-2 where the immunostaining increased for ICUD groups in all hippocampus areas, at all postischemia days, mainly in the CA3 area. Such effects were reverted in ICTD pup groups.

Arachidonic acid-derived lipid mediators are intimately involved in inflammation and biosynthesized by pathways dependent upon COX and LOX enzymes. The role of LOX and COX isoforms, particularly COX-2, in inflammation, is well established. At cellular and molecular levels, curcumin regulates a number of signaling pathways, including the eicosanoid pathway, involving COX and LOX [44]. An upregulation of COX-2 and iNOS was demonstrated in human infarcted brains, which agrees with the hypothesis that these enzymes and their reaction products contribute to the progression of postischemic cerebral injury [45]. Furthermore, curcumin is both a nitric oxide (NO) scavenger and an inhibitor of iNOS expression [46].

We showed that curcumin presents neuroprotective properties, in the model of global transient brain ischemia in rat pups, whose mothers were chronically administered with curcumin ( 25 and $50 \mathrm{mg} / \mathrm{kg}$, p.o.), since the 1st day of mating up to the end of lactation. It is important to notice that these doses are much lower than the ones used in the great majority of studies presented in the literature. In ischemic pups, we observed alterations in the contents of DA and its metabolite DOPAC, as well as cytoprotective actions that are dependent upon postischemic periods. Curcumin also decreases COX-2 and iNOS expressions upregulated in ischemic brains of pups. Considering that curcumin possesses anti-inflammatory and antioxidant actions, and the participation of inflammation and free radicals production in ischemic events, it is highly possible that these curcumin properties be involved with its neuroprotective activity, as demonstrated in the present work.

\section{Conclusions}

We may conclude that neurochemical and immunohistochemical alterations in the brain of ischemic pups can vary, according to the time elapsed after the ischemic insult. Furthermore, there is a strong possibility that the treatment of dams with low doses of curcumin reaches the fetuses, reversing the effects of ischemia in the pups, since the drug crosses the blood brain barrier and is detected in the CSF [18]. Curcumin biodisponibility problems, especially in humans, and those related to its biphasic dose-response curve are frequent. Thus, pharmaceutical formulations, as the recent developments of curcumin in nanoparticulate delivery systems [47], are urgently needed. This could minimize or even solve the biodisponibility problems and would certainly contribute to the inclusion of curcumin into the clinics, as a neuroprotective drug.

\section{Conflict of Interests}

The authors declare that there is no conflict of interests.

\section{Acknowledgments}

The authors are grateful to the financial support of the Brazilian National Research Council (CNPq), the Brazilian Ministry of Health (MS), and the Foundation for Technological and Scientific Development of the State of Ceará (FUNCAP). The authors also thank Professor M. O. L. Viana for his careful orthographic revision of the paper.

\section{References}

[1] Y.-C. Wang, S. Lin, and Q.-W. Yang, "Toll-like receptors in cerebral ischemic inflammatory injury," Journal of Neuroinflammation, vol. 8, article 134, 2011.

[2] S. J. Vannucci and H. Hagberg, "Hypoxia-ischemia in the immature brain," The Journal of Experimental Biology, vol. 207, no. 18, pp. 3149-3154, 2004.

[3] Y. K. Gupta, S. Briyal, and A. Gulati, "Therapeutic potential of herbal drugs in cerebral ischemia," Indian Journal of Physiology and Pharmacology, vol. 54, no. 2, pp. 99-122, 2010.

[4] C. Iadecola and J. Anrather, "The immunology of stroke: from mechanisms to translation," Nature Medicine, vol. 17, no. 7, pp. 796-808, 2011.

[5] R. Macrez, C. Ali, O. Toutirais et al., "Stroke and the immune system: from pathophysiology to new therapeutic strategies," The Lancet Neurology, vol. 10, no. 5, pp. 471-480, 2011.

[6] L. Garcia-Bonilla, C. Benakis, J. Moore, C. Iadecola, and J. Anrather, "Immune mechanisms in cerebral ischemic tolerance," Frontiers in Neuroscience, no. 8, article 44, 2014.

[7] A. Kuhad and K. Chopra, "Curcumin attenuates diabetic encephalopathy in rats: behavioral and biochemical evidences," European Journal of Pharmacology, vol. 576, no. 1-3, pp. 34-42, 2007.

[8] B. B. Aggarwal and K. B. Harikumar, "Potential therapeutic effects of curcumin, the anti-inflammatory agent, against neurodegenerative, cardiovascular, pulmonary, metabolic, autoimmune and neoplastic diseases," The International Journal of Biochemistry \& Cell Biology, vol. 41, no. 1, pp. 40-59, 2009. 
[9] C. Park, D.-O. Moon, I.-W. Choi et al., "Curcumin induces apoptosis and inhibits prostaglandin E2 production in synovial fibroblasts of patients with rheumatoid arthritis," International Journal of Molecular Medicine, vol. 20, no. 3, pp. 365-372, 2007.

[10] B. B. Aggarwal, A. Kumar, and A. C. Bharti, "Anticancer potential of curcumin: preclinical and clinical studies," Anticancer Research, vol. 23, no. 1, pp. 363-398, 2003.

[11] L. Xie, X.-K. Li, N. Funeshima-Fuji et al., "Amelioration of experimental autoimmune encephalomyelitis by curcumin treatment through inhibition of IL-17 production," International Immunopharmacology, vol. 9, no. 5, pp. 575-581, 2009.

[12] A. S. Darvesh, R. T. Carroll, A. Bishayee, N. A. Novotny, W. J. Geldenhuys, and C. J. van der Schyf, "Curcumin and neurodegenerative diseases: a perspective," Expert Opinion on Investigational Drugs, vol. 21, no. 8, pp. 1123-1140, 2012.

[13] D. S. Kim, J. Y. Kim, and Y. Han, "Curcuminoids in Neurodegenerative diseases," Recent Patents on CNS Drug Discovery, vol. 7, no. 3, pp. 184-204, 2012.

[14] A. Monroy, G. J. Lithgow, and S. Alavez, "Curcumin and neurodegenerative diseases," BioFactors, vol. 39, no. 1, pp. 122132, 2013.

[15] S. A. Frautschy, W. Hu, P. Kim et al., "Phenolic anti-inflammatory antioxidant reversal of $\mathrm{A} \beta$-induced cognitive deficits and neuropathology," Neurobiology of Aging, vol. 22, no. 6, pp. 9931005, 2001.

[16] F. Yang, G. P. Lim, A. N. Begum et al., "Curcumin inhibits formation of amyloid $\beta$ oligomers and fibrils, binds plaques, and reduces amyloid in vivo," The Journal of Biological Chemistry, vol. 280, no. 7, pp. 5892-5901, 2005.

[17] M. Garcia-Alloza, L. A. Borrelli, A. Rozkalne, B. T. Hyman, and B. J. Bacskai, "Curcumin labels amyloid pathology in vivo, disrupts existing plaques, and partially restores distorted neurites in an Alzheimer mouse model," Journal of Neurochemistry, vol. 102, no. 4, pp. 1095-1104, 2007.

[18] S. Mishra and K. Palanivelu, "The effect of curcumin (turmeric) on Alzheimer's disease: an overview," Annals of Indian Academy of Neurology, vol. 11, no. 1, pp. 13-19, 2008.

[19] S. K. Kulkarni and A. Dhir, "An overview of curcumin in neurological disorders," Indian Journal of Pharmaceutical Sciences, vol. 72, no. 2, pp. 149-154, 2010.

[20] J. M. Ringman, S. A. Frautschy, E. Teng et al., "Oral curcumin for Alzheimer's disease: tolerability and efficacy in a 24-week randomized, double blind, placebo-controlled study," Alzheimer's Research and Therapy, vol. 4, no. 5, article 43, 2012.

[21] A. Matteucci, R. Cammarota, S. Paradisi et al., "Curcumin protects against NMDA-induced toxicity: a possible role for NR2A subunit," Investigative Ophthalmology and Visual Science, vol. 52, no. 2, pp. 1070-1077, 2011.

[22] M. Thiyagarajan and S. S. Sharma, "Neuroprotective effect of curcumin in middle cerebral artery occlusion induced focal cerebral ischemia in rats," Life Sciences, vol. 74, no. 8, pp. 969$985,2004$.

[23] J. Zhao, Y. Zhao, W. Zheng, Y. Lu, G. Feng, and S. Yu, "Neuroprotective effect of curcumin on transient focal cerebral ischemia in rats," Brain Research, vol. 1229, pp. 224-232, 2008.

[24] A. Matteucci, C. Frank, M. R. Domenici et al., "Curcumin treatment protects rat retinal neurons against excitotoxicity: effect on N-methyl-D: -aspartate-induced intracellular $\mathrm{Ca}^{2+}$ increase," Experimental Brain Research, vol. 167, no. 4, pp. 641648, 2005.
[25] R. Wang, Y.-B. Li, Y.-H. Li, Y. Xu, H.-L. Wu, and X.-J. Li, "Curcumin protects against glutamate excitotoxicity in rat cerebral cortical neurons by increasing brain-derived neurotrophic factor level and activating TrkB," Brain Research, vol. 1210, pp. 84-91, 2008.

[26] N. W. Kleman, D. Sun, and P. Cengiz, "Mechanisms underlying neonatal hypoxia ischemia," The Open Drug Discovery Journal, vol. 2, no. 3, pp. 129-137, 2010.

[27] A. M. Comi, W. H. Trescher, R. Abi-Raad, M. V. Johnston, and M. A. Wilson, "Impact of age and strain on ischemic brain injury and seizures after carotid ligation in immature mice," International Journal of Developmental Neuroscience, vol. 27, no. 3, pp. 271-277, 2009.

[28] A. M. Comi, C. J. C. Weisz, B. H. Highet, M. V. Johnston, and M. A. Wilson, "A new model of stroke and ischemic seizures in the immature mouse," Pediatric Neurology, vol. 31, no. 4, pp. 254-257, 2004.

[29] R. Lim, G. Barker, C. A. Wall, and M. Lappas, "Dietary phytophenols curcumin, naringenin and apigenin reduce infectioninduced inflammatory and contractile pathways in human placenta, foetal membranes and myometrium," Molecular Human Reproduction, vol. 19, no. 7, pp. 451-462, 2013.

[30] S. Ganiger, H. N. Malleshappa, H. Krishnappa, G. Rajashekhar, V. Ramakrishna Rao, and F. Sullivan, "A two generation reproductive toxicity study with curcumin, turmeric yellow, in Wistar rats," Food and Chemical Toxicology, vol. 45, no. 1, pp. 64-69, 2007.

[31] R. Ramos-Zúñiga, P. U. Gómez, A. Navarro Ruiz, S. Luquín De A, and J. García-Estrada, "Locomotor activity is a predictive test after global ischemia-reperfusion in Mongolian gerbils," Minimally Invasive Neurosurgery, vol. 51, no. 2, pp. 87-90, 2008.

[32] H. Shen and Y. Wang, "Correlation of locomotor activity and brain infarction in rats with transient focal ischemia," Journal of Neuroscience Methods, vol. 186, no. 2, pp. 150-154, 2010.

[33] M. R. Milot and H. Plamondon, "Time-dependent effects of global cerebral ischemia on anxiety, locomotion, and habituation in rats," Behavioural Brain Research, vol. 200, no. 1, pp. 173180, 2009.

[34] K. Plaschke, M. Grant, M. A. Weigand, J. Züchner, E. Martin, and H. J. Bardenheuer, "Neuromodulatory effect of propentofylline on rat brain under acute and long-term hypoperfusion," British Journal of Pharmacology, vol. 133, no. 1, pp. 107-116, 2001.

[35] L.-W. Fan, S. Lin, Y. Pang, P. G. Rhodes, and Z. Cai, "Minocycline attenuates hypoxia-ischemia-induced neurological dysfunction and brain injury in the juvenile rat," European Journal of Neuroscience, vol. 24, no. 2, pp. 341-350, 2006.

[36] U. Ådén, L. Halldner, H. Lagercrantz, I. Dalmau, C. Ledent, and B. B. Fredholm, "Aggravated brain damage after hypoxic ischemia in immature adenosine $\mathrm{A}_{2 A}$ knockout mice," Stroke, vol. 34, no. 3, pp. 739-744, 2003.

[37] M. E. Haque, K.-I. Tanaka, and N. Ogawa, "Relationship between locomotor activity and monoamines following single and double transient forebrain ischemia in gerbils," Neurochemical Research, vol. 26, no. 4, pp. 401-406, 2001.

[38] F. Silverstein and M. V. Johnston, "Effects of hypoxia-ischemia on monoamine metabolism in the immature brain," Annals of Neurology, vol. 15, no. 4, pp. 342-347, 1984.

[39] J. Weinberger and J. Nieves-Rosa, "Metabolism of monoamine neurotransmitters in the evolution of infarction in ischemic striatum," Journal of Neural Transmission, vol. 69, no. 3-4, pp. 265-275, 1987. 
[40] M. Godinez-Rubi, A. E. Rojas-Mayorquin, D. Ortu, and D. Ortuño-Sahagun, "Nitric oxide donos as neuroprotective agentes after ischemic stroke-related inflammatory reaction," Oxidative Medicine and Cellular Longevity, vol. 2013, Article ID 297357, 16 pages, 2013.

[41] F. A. Al-Omar, M. N. Nagi, M. M. Abdulgadir, K. S. Al Joni, and A. A. Al-Majed, "Immediate and delayed treatments with curcumin prevents forebrain ischemia-induced neuronal damage and oxidative insult in the rat hippocampus," Neurochemical Research, vol. 31, no. 5, pp. 611-618, 2006.

[42] V. P. Menon and A. R. Sudheer, "Antioxidant and antiinflammatory properties of curcumin," Advances in Experimental Medicine and Biology, vol. 595, pp. 105-125, 2007.

[43] A. Goel, C. R. Boland, and D. P. Chauhan, "Specific inhibition of cyclooxygenase-2 (COX-2) expression by dietary curcumin in HT-29 human colon cancer cells," Cancer Letters, vol. 172, no. 2, pp. 111-118, 2001.

[44] Z. A. Radi and N. K. Khan, "Expression of COX-1, COX-2, iNOS and p38 in human brain with stroke lesions," International Journal of Pharmacology, vol. 4, no. 2, pp. 108-113, 2008.

[45] C. V. Rao, "Regulation of COX and LOX by curcumin," Advances in Experimental Medicine and Biology, vol. 595, pp. 213-226, 2007.

[46] M. Zheng, S. Ekmekcioglu, E. T. Walch, C.-H. Tang, and E. A. Grimm, "Inhibition of nuclear factor- $\kappa \mathrm{B}$ and nitric oxide by curcumin induces $\mathrm{G} 2 / \mathrm{M}$ cell cycle arrest and apoptosis in human melanoma cells," Melanoma Research, vol. 14, no. 3, pp. 165-171, 2004.

[47] G. Flora, D. Gupta, and A. Tiwari, "Nanocurcumin: a promising therapeutic advancement over native curcumin," Critical Reviews in Therapeutic Drug Carrier Systems, vol. 30, no. 4, pp. 331-368, 2013. 

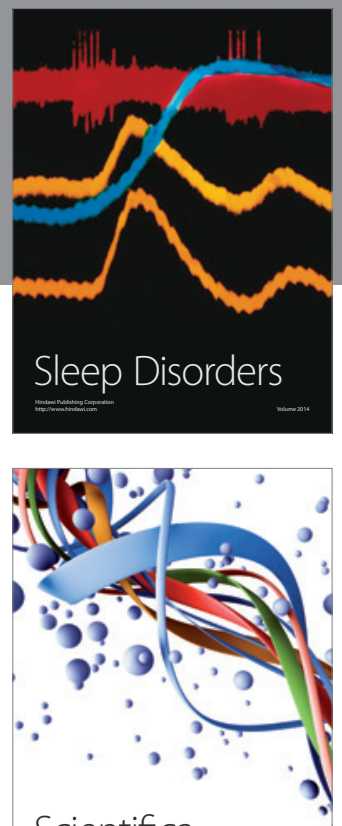

Scientifica
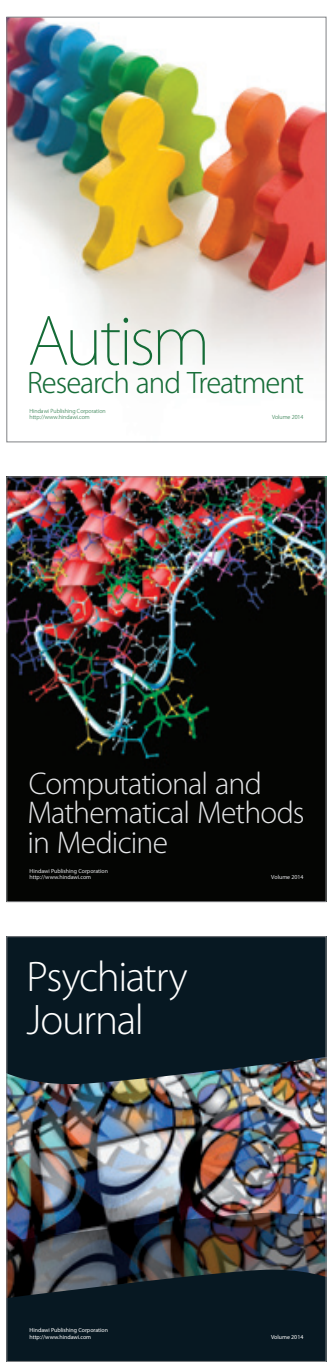
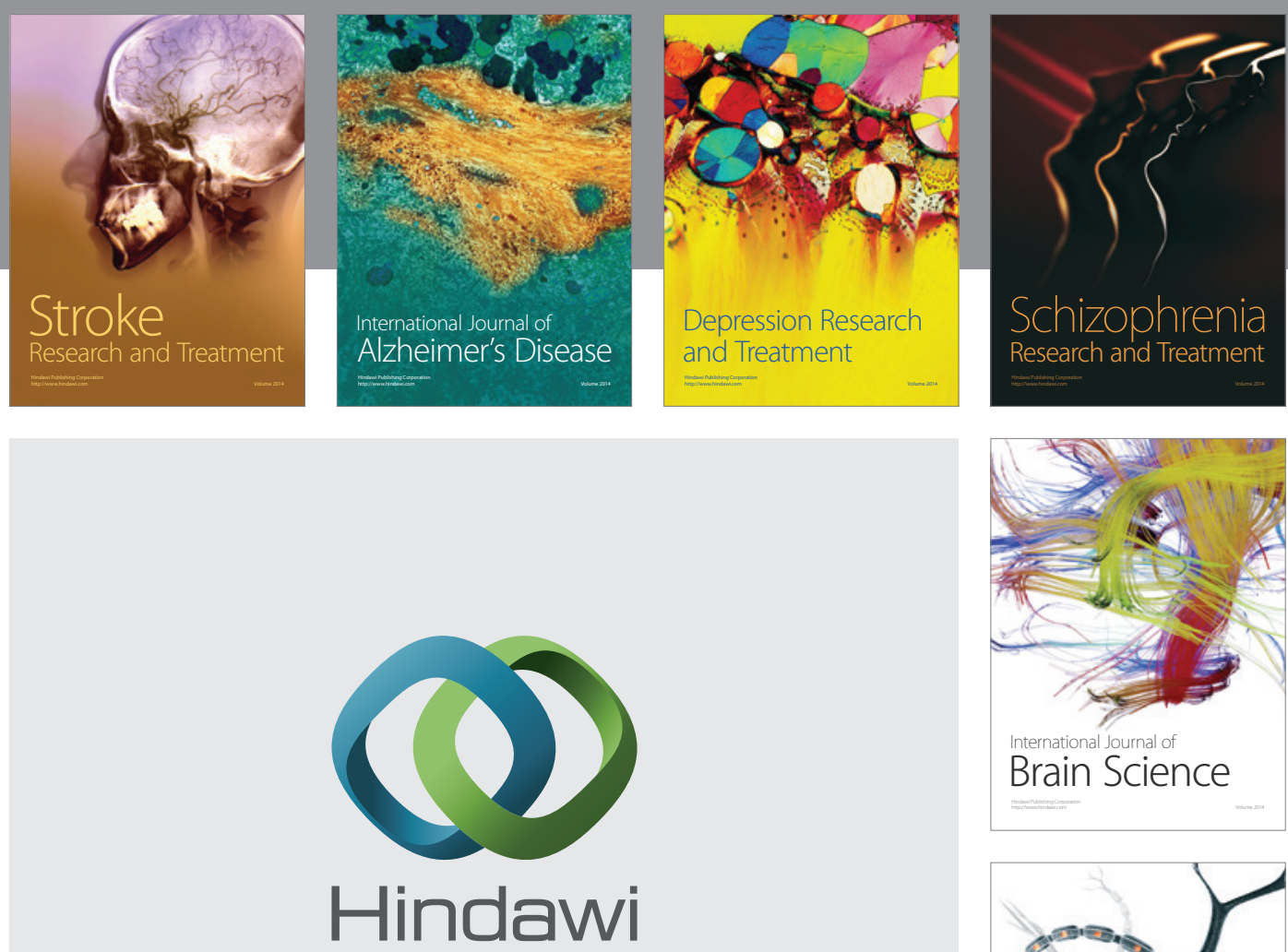

Submit your manuscripts at

http://www.hindawi.com
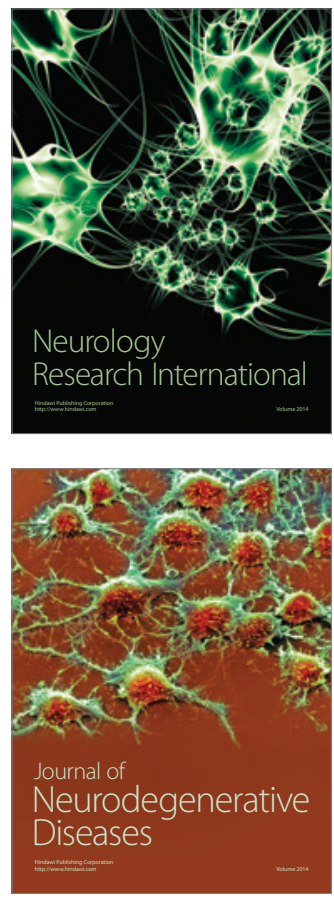

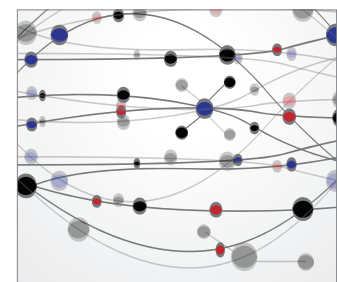

The Scientific World Journal
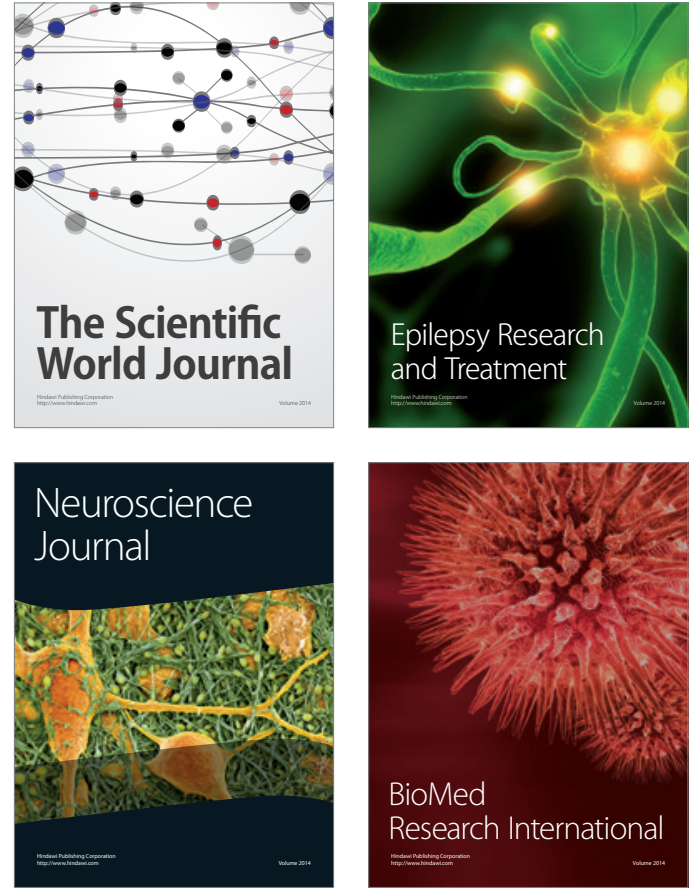

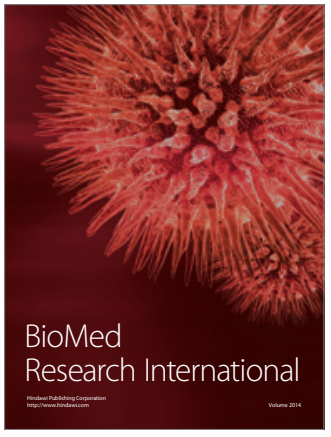

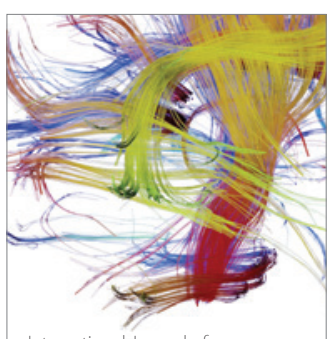

Brain Science

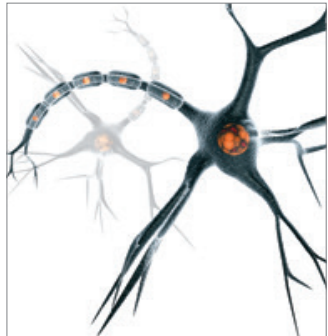

Neural Plasticity
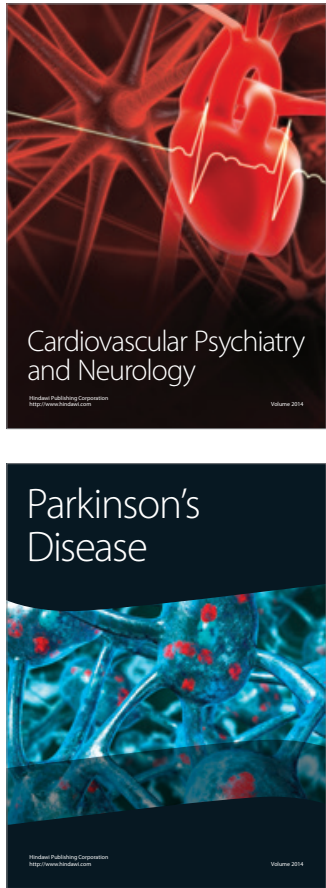